$\xi=-1$

\title{
Contrast Ratios, Colour Elements, and Polarities in Visual Acuity Measurements
}

\author{
Ai-Hong Chen*, Nurulain Muhamad \\ Optometry, Fakulti Sains Kesihatan, Universiti Teknologi Mara (UiTM), 42300 Bandar Puncak Alam, Selangor, Malaysia \\ *Corresponding author E-mail: chenaihong@salam.uitm.edu.my
}

\begin{abstract}
This study was aimed to investigate the effects of contrast ratios, colour elements, and polarities on visual acuity measurements. The visual acuities of 18 subjects were measured using 6 different modifications of the Landolt C chart in a 4-metre LogMAR design: (M1) black-on-white [contrast ratio: 0.8]; (M2) white-on-black [contrast ratio: 0.8]; (M3) black-on-white [contrast ratio: 0.5]; (M4) yellow-ongreen [contrast ratio: 0.8]; (M5) yellow-on-green [contrast ratio: 0.5]; and (M6) black-on-yellow [contrast ratio: 0.8]. Comparative analysis was performed to examine the effects of the three aforementioned parameters on visual acuity measurements. The visual acuities were found to be significantly different between the contrast ratios of 0.5 and 0.8 , regardless of whether colour elements were present (blackon-white) or otherwise (yellow-on-green) [Friedman test: $\chi^{2}(3)=24.24, p<0.05$ ]. Interestingly, the LogMAR score was found to be significantly different between the yellow-on-green and black-on-yellow chart designs when the contrast ratio remained constant at 0.8 [Wilcoxon signed-rank test: $\mathrm{z}=2.950, \mathrm{p}<0.05$ ]. However, the effect of contrast polarity (positive/ negative) on visual acuity was not significant when the contrast ratio was fixed at 0.8 [Wilcoxon signed-rank test: $\mathrm{z}=0.318, \mathrm{p}>0.05$ ]. A lower contrast ratio and midrange wavelength colour reduced visual acuity. The effect of contrast polarity without colour elements on visual acuity was negligible.
\end{abstract}

Keywords: Contrast Polarity; Contrast Ratio; Colour; Visual Acuity

\section{Introduction}

Visual acuity is used to assess visual functions, especially in clinical settings [1]. Over the past centuries, visual acuity has been studied, measured and analysed because it represents a fundamental limit in human visual system to see. It measures the resolving capacity of human visual system. Consequently, it has widely been used in a criterion to determine whether the person meet occupational vision standards. In addition, visual acuity has also become as an indicator of stability of pathological conditions [2]. Visual acuity is classified into four broad groups; visual detection, visual recognition, visual discrimination and visual resolution. These visual acuity classifications are representing different limits and may be determined by different aspects of the visual pathways. In visual detection, it represents the minimum visible acuity that one can detect [1]. It is determined by the difference between brightness of an object against its background. Visual detection measures threshold size of a spot required to detect its presence against its background. Hence, generally it is a very gross state of measuring the visual acuity. The ability to recognize or identify smallest angular size is measured in visual recognition [3]. Normally, in clinical setting, this is achieved by utilizing optotypes or shapes. In this method, the visual recognition is measured through a progressively smaller optotypes. The visual discrimination is measured by the ability to discriminate changes in the angular size of the minimal change in the attribute [1]. This involves changes in the physical features of the object such as size, orientation and positions. Minimum discriminable ability is also known as the hyperacuity or vernier acuity. The fourth group of visual function is the visual resolution. Visual resolution represents the ability to perceive a minimum resolvable or minimum separable of an object [4]. It is a spatial discrimination of visual threshold. In this study design, the visual resolution was employed in the measures of visual function system. There are numerous factors that can influence the ability of visual system to resolve a fine detail.

The corrected refractive error of visual acuity test is the gold standard routine assessment in optometry and ophthalmology. The Snellen's chart is widely used for evaluating visual acuity. However, the progression between each line in the chart is irregular and hence, inadequate to detect visual acuity changes in terms of the number of lines which the subject is able or unable to see [1], [5]. As such, in the latest studies on vision, the Bailey-Lovie acuity chart and the Early Treatment Diabetic Retinopathy Study (ETDRS) charts, which employ the logMAR progression (five numbers or letters in each line, with equal heights and widths for each character), are most preferred [5], [6]. Chart designs are based on orientations. For example, Landolt $\mathrm{C}$ or Illiterate $\mathrm{E}$ are good alternatives when the legibility of different letters are a concern [7]. The resolution of an optotype or letter is dependent on its contrast [8]-[10].

In a real world, ability to function (vision) in multicontrast environment is important as it affects the quality of life. Contrast is not merely dependent to the differences in luminance between two adjoining areas. There is however other factors as well that contributes to the contrast parameters. In certain conditions where both luminance and colour are less effective and conspicuous such as in when colour discrimination was inhibited in low light environments, contrast polarity might contribute to visual response enhancement. Previous studies on polarity and contrast only had 
small sample sizes, so it was difficult to draw strong conclusions [11]-[14]. The demand for the contrast-detecting component of visual resolution has been reported to decrease when the contrast of the visual acuity chart was reversed (i.e. white-on-black) [13]. The reduction is mainly due to the changes in the contrast of the features which occur in the retina. It has been hypothesised that the resolutions of reversed-contrast polarity charts differ from that of the traditional charts. The effect of contrast polarity on visual acuity has been investigated by comparing the outcomes of the usage of conventional black-on-white charts and reversed-contrast charts [13], [14]. Evidently, the logarithm of the minimum angle of resolution (LogMAR) was significantly smaller for white letters on a dark background [13]. However, the study had a small sample size (4 subjects) and did not control the learning effect (constant stimuli were used when measuring the visual acuity, so guessing or biases might have led to inaccurate results) [15]. Also, the reports on polarity were inconclusive. Some claimed that negative polarity was preferred for better legibility [11], [12], while others believed that contrast polarity did not affect to visual acuity [16].

Visual acuity reduces when the contrast ratio is low [10], [17], [18]. In a study, the reduction was greater when low- (0.14) and medium-contrast $(0.21)$ charts were used instead of high-contrast (0.88) charts [17]. However, the value of the medium contrast used in the study was relatively lower as compared those of other studies [10], [19]. Previous studies on the functional roles of contrast adaptation have found discrepancies and inconsistencies in the discrimination threshold for background contrast ratios of 0.1 -0.4 [20]. Meanwhile, a high level of agreement was found when the contrast ratio was between $0.5-0.8$, with the greatest improvement in the discrimination threshold occurring when the contrast ratio was 0.8 . Hence, it was suggested that the discrimination threshold for background contrast was influenced by medium to high contrast ratios.

Most of the investigations have employed the high contrast ratio predominantly in contrast sensitivity test, whereby the contrast was reduced according to a greyscale pattern [21]. Generally, the contrast sensitivity test is considered to be more accurate reflect visual function in daily life as real world have various luminance contrast [22]-[24]. However, the contrast sensitivity test has its limit, in which it does not provide colour information. The rationale of incorporating colour information in other visual function test (such as visual acuity) could be useful for evaluating the quality of vision in daily life. This is because human visual perception is made up of spatial vision, temporal vision and colour vision [15]. However, previous studies on the effect of colour elements on visual performance, especially during reading, did not provide detailed quantitative information regarding the colours which were used as stimuli [25]-[27]. Therefore, the results could have been difficult to be interpreted scrupulously with respect to the effects of colours and contrasts. Studies on different text and background colour combinations have noted that unlike lowcontrast combinations, high-contrast ones were rated more favourably during subjective evaluations, apart from resulting in better performance [28]. Hence, the interactions between contrast polarities, contrast ratios, and colour elements in visual performance require further investigations.

Our study has investigated the effects of three independent variables (contrast ratios, colour elements and polarities) on visual acuity (dependent variable) using LogMAR scores. It is hypothesized that the dependency of visual acuity is equally effectuated by contrast ratios, colour elements and polarities.

\section{Materials and Methods}

\subsection{Visual Stimuli}

Visual acuity measurements were carried out in a room of dimensions $7.4 \mathrm{~m}$ (length) $\times 3 \mathrm{~m}$ (width) $\times 3 \mathrm{~m}$ (height). The measure- ments were taken for each of the 6 different modifications of the printed Landolt $\mathrm{C}$ (see Fig.1) chart in a 4 metre LogMAR designs:

1. (M1) black-on-white (contrast ratio: 0.8)

2. (M2) white-on-black (contrast ratio: 0.8)

3. (M3) black-on-white (contrast ratio: 0.5)

4. (M4) yellow-on-green (contrast ratio: 0.8 )

5. (M5) yellow-on-green (contrast ratio: 0.5 )

6. (M6) black-on-yellow (contrast ratio: 0.8)

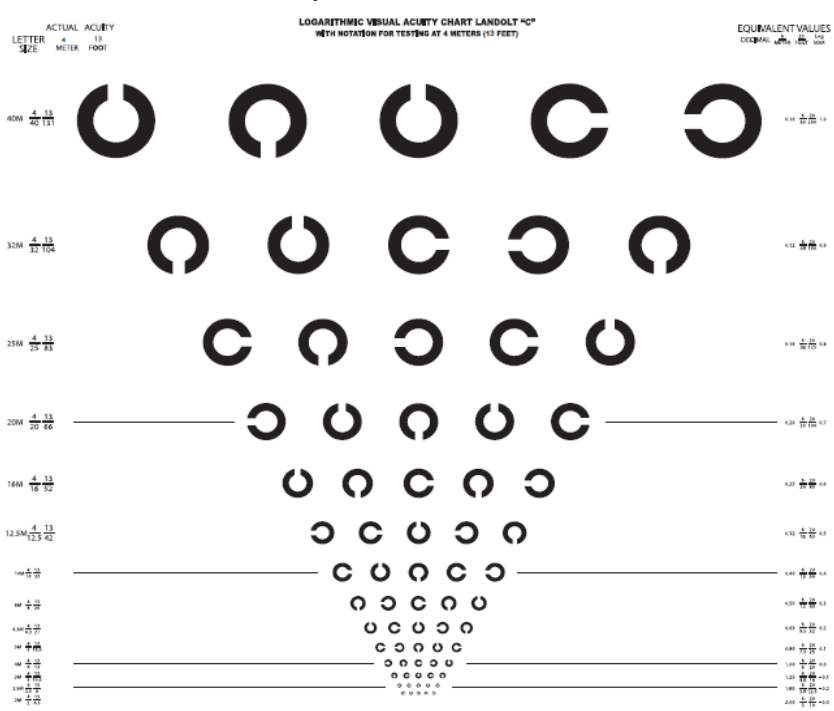

Fig. 1: Landolt $C$ chart

The luminances of the charts were measured by a Konica Minolta Luminance Meter LS110. The averages of three measurements were taken as the contrast values. The luminance contrast definition of Michelson was used to calculate the contrast ratio and this was based on maximum and minimum luminance of the text and the background [15]. The luminance was tested at $120 \mathrm{~cd} / \mathrm{m}^{2}$, which was within the recommendation for the luminance of standardised charts to be between $85 \mathrm{~cd} / \mathrm{m}^{2}$ and $300 \mathrm{~cd} / \mathrm{m}^{2}$ [29]. A fourorientation Landolt $\mathrm{C}$ chart design was introduced to the subjects [30] with an internally illuminated by standardised light-emitting diodes (LED) lamp that known for its lesser flicker [31], [32] and provides better colour properties [33].

\subsection{Procedure}

The subjects were seated on an adjustable ergonomic chair, with the Landolt $\mathrm{C}$ chart positioned $4 \mathrm{~m}$ directly ahead. Six Landolt $\mathrm{C}$ charts of different designs (M1 - M6) were presented to the subjects at random. The subjects were light-adapted for two minutes in the same experimentation room. The reason of this was to allow the regeneration of sufficient cone pigments to detect the luminance of the charts [34]. Hence, it was important to ensure that the photoreceptors remained sensitive to the luminance of the charts before the visual acuity measurements were taken. A 'criterionfree' method was employed, so that the results were not affected by the cautiousness of the subjects' responses. The visual acuities were measured in terms of the logarithm of the minimum angle of resolution (LogMAR) at which the gap orientations of the Landolt $\mathrm{C}$ chart were accurately identified. The scoring was based on the letter-by-letter termination rule, for which the subjects were encouraged to complete a particular line. Scoring acuity letter-byletter, in which equal credit is given for each correct letter read, produced better test-retest variability and finer grading scale [35]. The visual acuities of the subjects were determined binocularly.

\subsection{Participants}

A total of eighteen subjects have been recruited. The sample size calculation was done using the GPower Analysis Version 3.1.9.2 
software, with a power of 0.95 . The inclusion criteria were (i) a corrected visual acuity of $6 / 6$ or higher in both eyes, (ii) subjects who have passed the Farnsworth D15 colour vision screening test, and (iii) an absence of known ocular diseases. Ethical approval was obtained from the Research Ethics Committee of Universiti Teknologi MARA. The series of comparisons in our study are summarised in Table 1.

Table 1: Summary of comparison in our study

\begin{tabular}{|c|c|}
\hline Objectives & Comparison \\
\hline $\begin{array}{l}\text { To compare positive and negative po- } \\
\text { larity using fixed contrast ratio at } 0.8\end{array}$ & $\begin{array}{l}\text { M1 (black-on-white) and M2 } \\
\text { (white on black) }\end{array}$ \\
\hline $\begin{array}{c}\text { To compare two different contrast ratio } \\
(0.8 \text { versus } 0.5) \text { using positive polarity } \\
\text { only }\end{array}$ & $\begin{array}{l}\text { M1 (black-on-white, } 0.8 \text { con- } \\
\text { trast ratio) and M3 (black-on- } \\
\text { white, } 0.5 \text { contrast ratio) }\end{array}$ \\
\hline $\begin{array}{l}\text { To compare the mean visual acuity } \\
\text { difference with and without colour } \\
\text { element as a result of the } 0.3 \text { contrast } \\
\text { ratio difference. }\end{array}$ & $\begin{array}{l}\text { Set without colour element: } \\
\text { M1 (black-on-white, } 0.8 \text { con- } \\
\text { trast ratio) and M3 (black-on- } \\
\text { white, } 0.5 \text { contrast ratio) } \\
\text { Set with colour element: } \\
\text { M4 (yellow-on-green, } 0.8 \\
\text { contrast ratio) and M5 (yellow- } \\
\text { on-green, } 0.5 \text { contrast ratio) }\end{array}$ \\
\hline $\begin{array}{l}\text { To compare the mean visual acuity } \\
\text { difference with and without colour } \\
\text { element at fixed } 0.8 \text { contrast ratio. }\end{array}$ & $\begin{array}{l}\text { M4 (yellow-on-green, } 0.8 \\
\text { contrast ratio) and M6 (black- } \\
\text { on-yellow, } 0.8 \text { contrast ratio). }\end{array}$ \\
\hline
\end{tabular}

\section{Results and Discussions}

The data was not normally distributed (Shapiro-Wilk: $\mathrm{p}<0.05$ ). Nonparametric tests such as the Wilcoxon signed-rank test and Friedman test were used. The level of significance was set at 0.05 . Comparative analyses were performed to examine the effects of contrast polarities, contrast ratios, and colour elements on visual acuity (see Fig.2).

\subsection{Fixed Contrast Ratio: Positive and Negative Polari- ty}

The comparison of visual acuity between positive and negative polarity was determined using the Wilcoxon signed-rank test. The LogMAR scores were not significantly different between the positive contrast polarity, M1 (median $=-0.06 ; \mathrm{IQR}=0.10$ ) and nega-

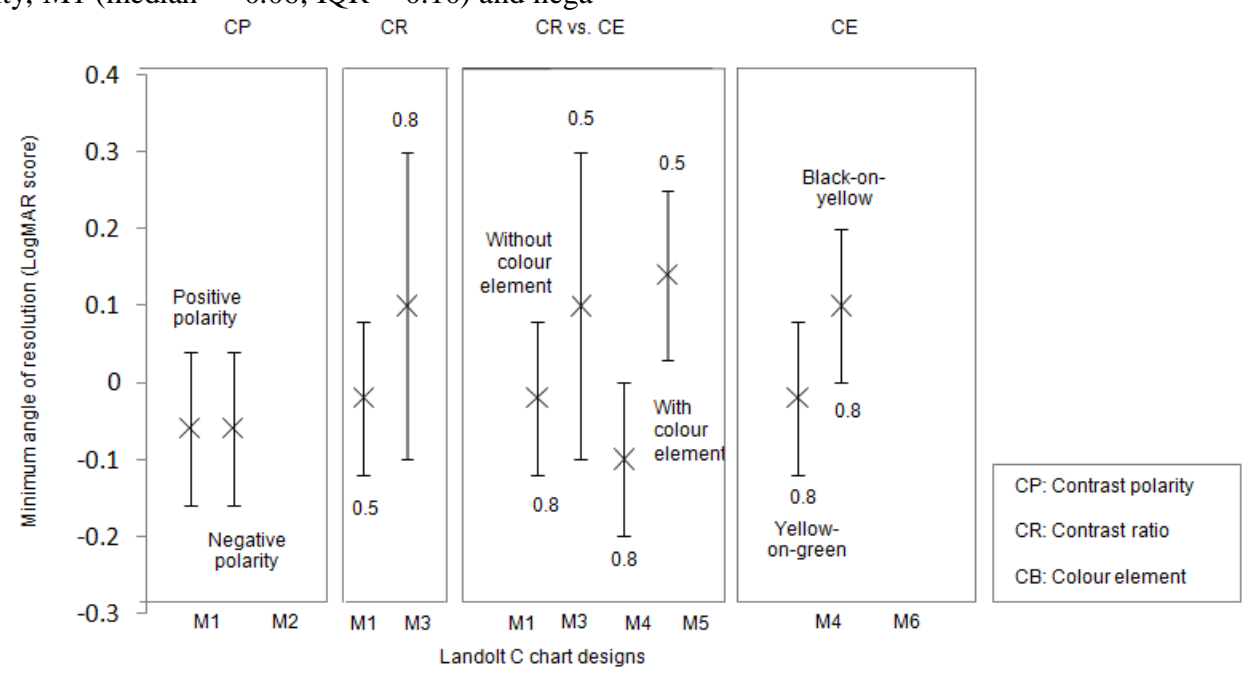

Fig. 2: The effect of contrast polarity, contrast ratio and colour element on visual acuity measurements tive contrast polarity, $\mathrm{M} 2$ (median $=-0.06 ; \mathrm{IQR}=0.10)$ [Wilcoxon signed-rank test: $\mathrm{z}=0.318, \mathrm{p}>0.05]$.

\subsection{Varied Contrast Ratio: Positive Polarity}

Visual acuity was further investigated in the presence of positive polarity and a contrast ratio of either 0.5 or 0.8 . The LogMAR score was significantly reduced when the contrast ratio was 0.5 (median $=0.10 ; \mathrm{IQR}=0.22$ ) as compared to the contrast ratio of 0.8 (median $=-0.02 ; \mathrm{IQR}=0.10$ ) [Wilcoxon signed-rank test: $\mathrm{z}=$ $2.944, \mathrm{p}<0.05]$.

\subsection{Varied Contrast Ratio: with and without Text/ Background Colour Elements}

The contrast ratios of four Landolt $\mathrm{C}$ chart designs were studied with and without colour elements. There were significant differences between the groups [Friedman test: $\chi^{2}(3)=24.24, p<$ 0.05]. Pairwise comparisons were performed using Dunn's procedure, with Bonferroni correction for multiple comparisons. The adjusted p-values were then presented. Post-hoc analyses showed significant differences in the LogMAR scores between the blackon-white chart with a contrast ratio of $0.8, \mathrm{M} 1$ (median $=-0.02$; $\mathrm{IQR}=0.10)$ and the black-on-white chart with a contrast ratio of 0.5 , M3 (median $=0.10 ; \mathrm{IQR}=0.22)(\mathrm{p}<0.05)$. Significant differences were also found between the yellow-on-green chart with a contrast ratio of $0.8, \mathrm{M} 4$ (median $=-0.10 ; \mathrm{IQR}=0.10)$ and the yellow-on-green chart with a contrast ratio of 0.5 , M5 (median = $0.14 ; \mathrm{IQR}=0.10)(\mathrm{p}<0.05)$. Additionally, the median visual acuity comparison with and without colour element as a result of 0.3 contrast ratio difference was found to be significant [Wilcoxon signed-rank test: $\mathrm{z}=2.944, \mathrm{p}<0.05$ for no colour element; $\mathrm{z}=$ $2.852, \mathrm{p}<0.05$ for colour element].

\subsection{Fixed Contrast Ratio: with and without Text Colour Elements}

The effect of colour element was further investigated at a contrast ratio at 0.8 . Interestingly, the yellow-on-green chart (i.e. text colour elements present), M4 (median $=-0.02 ; \mathrm{IQR}=0.10)$ gave better visual acuity measurements than the black-on-yellow chart (i.e. text colour elements absent), M6 (median $=0.10 ; \mathrm{IQR}=0.10$ ) [Wilcoxon signed-rank test: $\mathrm{z}=2.950, \mathrm{p}<0.05$ ]
Contrast ratios and colour elements significantly influenced the visual acuity outcomes in our study, but this effect was not apparent in different contrast polarities. Our finding seemed to disagree with previous reports on the improvement of visual acuity in the presence of negative polarity [13], [14]. However, the methodological approaches in the latter were different. First, 
previous investigations have utilised non-printed materials and constant stimuli method. On the other hand, our study has adopted printed materials and 'criterion-free' forced-choice method. Constant stimuli approaches tend to give a wider range of measurements due to the subjective interpretations of blurriness and clearness [15]. The forced-choice method in our study could have reduced the effects of subjective interpretations because the observers' judgments were not absolute; they were comparative instead [36]. Hence, it is suggested that the effects of the observers' perceptions towards the measurement were minimal. Second, a testing distance of $1.9 \mathrm{~m}$ in previous studies could have induced more reflex accommodative blurriness than the testing distance of 4 $\mathrm{m}$ in our study. Reflex accommodation is an automatic ocular response to blurriness in an attempt to maintain a clear retinal image. In addition, the target on a computer screen in a dimly-lit room in the previous studies could have been a source of glare to the subjects, which in turn affected the visual acuity measurements. Previous studies have claimed that negative polarity provides better legibility as compared to positive polarity [12]. However, the black-on-white (positive polarity) and white-on-black (negative polarity) analyses have not been conducted in isolation (i.e. without the influence of other colours). Hence, the precise effect of contrast polarity without colour elements on visual acuity was difficult to determine. A similar finding was reported in an investigation which employed 56 colour combinations in a liquid crystal display (LCD) [11]. On another note, contrast polarity, be it black-on-white (positive polarity) or white-on-black (negative polarity), in a visual display terminal was reported to have no significant effects on visual performance [16]. In other words, the mean visual acuity was not affected by the contrast polarity. Our findings revealed similar trends, although we used a different type of visual stimulus (i.e. printed materials). In our study, no significant difference in the visual acuity has been noted between the positive and negative polarities without the presence of colour elements.

Visual acuity decreased as the contrast ratio decreased. In our study, a similar pattern was observed in both with the presence and absence of colour elements. A lower contrast ratio (0.5) gave poorer LogMAR scores than a higher contrast ratio (0.8). In an earlier study on the effects of contrast ratios on visual acuity, analyses of variances showed that the latter was significantly affected by the former [16]. There was a significant increment in the visual acuity at higher contrast ratios. Our finding was consistent with that of a study in which at a contrast ratio of 0.8 , both the presence and absence of colour elements yielded better visual acuities of -0.02 and -0.10 respectively, as compared to a contrast ratio of 0.5 . These results matched those of earlier studies, in which the greatest improvement in the discrimination threshold was noted when the background contrast level was at its highest (i.e. 0.8) [20]. Interestingly, the reduction of the LogMAR score which was caused by a decrease in the contrast ratio seemed to be greater when colour elements were present in the Landolt $\mathrm{C}$ chart design. Our findings have managed to confirm that colour combinations with higher contrast were better than those with lower contrast [28]. It is somewhat surprising that the colour combination of yellow-ongreen produced better LogMAR score compared to black-onyellow despite of both charts having same contrast ratio. This finding was contradicted with earlier study that has found poor visual acuity with the colour combination of green-yellow [37]. This inconsistency may be due to utilization of the Landolt $\mathrm{C}$ text/background designs used in both studies. Our study utilized a single yellow colour as the text and background while the earlier study mixed both yellow and green colours as the text. Indirectly, this suggests that simultaneous contrast that was produced due to colour differences between the text and background play a significant role and the effect was apparent in yellow-on-green chart design. This finding further support the idea of changeable character of green colour when being used as the background [38]. Radiant effect was obtained with yellow on green, with yellow dominating over the green [38]. This occurred as green is a mixture of two complementary colours (yellow and blue), as compared to yellow which is the complementary colour. The interaction between colours in the simultaneous contrast is especially perceptible when objects have complementary colours [39]. Overall, when colour elements were present, regardless of whether the contrast was reduced or that the colour was just applied as a background, the visual resolution degraded. Colour elements seemed to affect the contrast mechanism in the ocular pathway. This was based on the finding that the same difference in the contrast ratio gave different results in the presence and absence of colour elements.

Although the study has reached its aims, there are some limitations and short comings. There are several limitations in this study which may need improvement in the future. This study drew its sample from a relatively small group of young adults (mean age of $22 \pm$ 1.81 years, $\mathrm{n}=18$ ). Therefore, it might not be suitable to generalize the results for pediatric or aging population. Further studies with larger sample size are needed to evaluate in normal population. Indeed, it would be interesting to assess any age-related changes of coloured visual acuity in the future study. Furthermore, we did not consider the effect of the refractive error since all subjects were screened for visual acuity of $6 / 6$ or better. The findings could be more interesting if habitual visual acuity is considered as subject inclusion criteria.

\section{Conclusion}

Returning to the hypothesis posed at the beginning of this study, it is now possible to state that the colour combinations of the targets and backgrounds of any visual function tests should be applied with caution and need further investigations. For any visual task involving the resolution and recognition of low colour contrast stimuli, deterioration of the visual resolution might occur. Hence, future investigations should incorporate a broader spectrum of colours which cover a wider range of wavelengths. In conclusion, the effect of polarity on visual acuity was negligible without the interference of contrast ratios and colour elements. Lower contrast ratios and colour elements reduced visual acuity. Of the three evaluated factors, the effect of colour combinations on visual acuity remains inconclusive and therefore requires further investigations.

\section{Acknowledgement}

This work was financially supported by the Universiti Teknologi MARA [Project Number: 600-IRMI/MYRA 5/3/LESTARI (K) $(172 / 2017)]$.

\section{References}

[1] C. Kniestedt and R. L. Stamper, "Visual acuity and its measurement," Ophthalmol. Clin. North Am., vol. 16, pp. 155$170,2003$.

[2] M. P. Nadler, D. Miller, and D. Nadler, Glare and Contrast Sensitivity for Clinicians. 1990.

[3] D. M. Levi, "Visual acuity," in Adler's Physiology of The Eye, Eleventh E., L. A. Levin, S. F. E. Nilsson, J. Ver Hoeve, and S. M. Wu, Eds. Elsevier Inc., 2011, pp. 627-647.

[4] I. L. Bailey, "Visual acuity," in Borish's Clinical Refraction, Second Edi., Butterworth Heinemann, 2006, p. 217.

[5] F. L. Ferris, A. Kassoff, G. H. Bresnick, and I. Bailey, "New Visual Acuity Charts for Clinical Research," Am. J. Ophthalmol., vol. 94, no. 1, pp. 91-96, Jul. 1982.

[6] I. L. Bailey and J. E. Lovie, "New Design Principles for Visual Acuity Letter Charts," Am. J. Optom. Physiol. Opt., vol. 53, no. 11, pp. 740-745, 1976

[7] J. S. Husk and D. Yu, "Learning to recognize letters in the periphery: Effects of repeated exposure, letter frequency, and letter complexity," J. Vis., vol. 17, pp. 1-11, 2017.

[8] F. W. Campbell and D. G. Green, "Optical and retinal factors affecting visual resolution," J. Physiol, vol. 181, pp. 576-593, 1965.

[9] I. Lie, "Visual detection and resolution as a function of adaptation and glare," Vision Res., vol. 21, no. 12, pp. 17931797,1981 
[10] D. Regan and D. Neima, "Low-contrast letter charts as a test of visual function.," Ophthalmology, vol. 90, no. 10, pp. 1192$1200,1983$.

[11] I. Humar, M. Gradisar, T. Turk, and J. Erjavec, "The impact of color combinations on the legibility of text presented on LCDs," Appl. Ergon., vol. 45, pp. 1510-1517, 2014.

[12] I. Humar, M. Gradisar, T. Turk, and J. Erjavec, "The impact of color combinations on the legibility of a Web page text presented on CRT displays," Appl. Ergon., vol. 38, pp. 885-899, 2008.

[13] G. Westheimer, "Visual Acuity with Reversed-Contrast Charts : I. Theoretical and Psychological Investigations," Optom. Vis. Sci., vol. 80, no. 11, pp. 745-748, 2003.

[14] G. Westheimer, P. Chu, W. Huang, T. Tran, and R. Dister, "Visual Acuity with Reversed-Contrast Charts: II. Clinical Investigation," Optom. Vis. Sci., vol. 80, no. 11, pp. 749-752, 2003.

[15] S. H. Schwartz, Visual Perception A Clinical Orientation, 4th Editio. New York: McGraw-Hill, 2010.

[16] A. Wang and M. Chen, "Effects of polarity and luminance contrast on visual performance and VDT display quality," Int. J. Ind. Ergon., vol. 25, pp. 415-421, 2000.

[17] A. Ho and S. M. Bilton, "Low contrast charts effectively differentiate between types of blur," Am. Acad. Optom., vol. 63, no. 3, pp. 202-208, 1986.

[18] P. R. Herse and H. E. Bedell, "Contrast Sensitivity for Letter and Grating Targets under Various Stmulus Conditions," Optom. Vis. Sci., vol. 66, no. 11, pp. 774-781, 1989.

[19] E. M. Mowry et al., "Vision related quality of life in multiple sclerosis: correlation with new measures of low and high contrast letter acuity," J. Neurol. Neurosurgery, Psychiatry, vol. 80, no. 7, pp. 767-772, 2009.

[20] M. W. Greenlee and F. Heitger, "The functional role of contrast adaptation*," Vision Res., vol. 28, no. 7, pp. 791-797, 1988.

[21] G. H. Portnoy, J. Brabyn, M. E. Schneck, and A. Jampolsky, "The SKILL Card: An Acuity Test of Reduced Luminance and Contrast," Investig. Opthalmology Vis. Sci., vol. 38, no. 1, pp. 207-218, 1997.

[22] L. T. Chylack et al., "Contrast sensitivity and visual acuity in patients with early cataracts," J. Cataract Refract. Surg., vol. 19, no. 3, pp. 399-404, 1993.

[23] S. Rubin, I. A. Adamsons, and W. J. Stark, "Comparison of Acuity , Contrast Sensitivity, and Glare Disability Before and After Cataract," Arch. Ophthalmol., vol. 111, pp. 56-61, 1993.

[24] D. B. Elliott, M. a Hurst, and J. Weatherill, "Comparing clinical tests of visual function in cataract with the patient's perceived visual disability.," Eye London Engl., vol. 4, no. 5, pp. 712-717, 1990.

[25] K. Knoblauch, A. Arditi, and J. Szlyk, "Effects of chromatic and luminance contrast on reading," J. Opt. Soc. Am. A, vol. 8, no. 2, p. 428 , Feb. 1991

[26] G. E. Legge, D. H. Parish, A. Luebker, and L. E. E. H. Wurm, "Psychophysics of Reading: XI . Comparing Color Contrast and Luminance Contrast," J. Opt. Soc. Am. A, vol. 7, no. 10, pp. 2002-2010, 1990.

[27] M. A. Tinker and D. G. Paterson, "Studies of typographical factors influencing speed of reading VII. Variations in color of print and background," pp. 246-251, 1931.

[28] J. Ling and P. van Schaik, "The effect of text and background colour on visual search of Web pages," Displays, vol. 23, pp. 223-230, 2002.

[29] International Council of Ophthalmology, "Visual Acuity Measurement Standard," Ital. J. Ophthalmol., vol. 2, no. 1, pp. $1-15,1988$

[30] M. Schrauf and C. Stern, "The visual resolution of Landolt-C optotypes in human subjects depends on their orientation: The 'gap-down' effect," Neurosci. Lett., vol. 299, pp. 185-188, 2001.

[31] P. Fang and Y. F. Liu, "Energy Channeling LED Driver Technology to Achieve Flicker-Free Operation with True Single Stage Power Factor Correction," IEEE Trans. Power Electron., vol. 32, no. 5, pp. 3892-3907, 2017.

[32] R. L. dos. Santos, D. M. Rufino, M. B. M. de Morais, and E. M. $\mathrm{Sa}$, "A charge-pump led driver with PFC and low-frequencyflicker reduction," 2017 Brazilian Power Electron. Conf., pp. 17, 2017.

[33] P. R. Boyce, Human Factors in Lighting, 3rd Editio. Taylor \& Francis Group, 2014.

[34] Rachel V. North, "Visual performance," in Work and the Eye, Second Edi., Butterworth Heinemann, 2001, pp. 1-17.

[35] I. L. Bailey, M. A. Bullimore, T. W. Raasch, and H. R. Taylor,
"Clinical grading and the effects of scaling," Investig. Ophthalmol. Vis. Sci., vol. 32, no. 2, pp. 422-432, 1991.

[36] K. E. Hinggins, M. J. Jaffe, N. J. Coletta, and R. C. Caruso, "Spatial Contrast Sensitivity Importance of Controlling the Patient â $€^{\mathrm{TM}}$ s Visibility Criterion," Arch Ophthalmol, vol. 102, pp. 1035-1041, 1984.

[37] Y. Tanaka et al., "Effect of Background Luminance Level on the Assessment of Color Visual Acuity Using Colored Landolt Rings in Young Healthy Subjects," Curr. Eye Res., vol. 43, no. 3, pp. 428-434, 2018.

[38] J. Itten, The art of color. Augsburg: Seiler \& Jehle, 1973.

[39] J. Albers, Interaction of color: Revised and expanded edition. New Haven, CT: Yale University Press, 2006. 